EPJ Web of Conferences 70, 00024 (2014)

DOI: $10.1051 /$ epjconf/ 20147000024

(C) Owned by the authors, published by EDP Sciences, 2014

\title{
New States with Heavy Quarks
}

\author{
Marek Karliner ${ }^{1, a}$, Harry J. Lipkin ${ }^{2,3, b}$, and Nils A. Törnqvist ${ }^{4, c}$ \\ ${ }^{1}$ Raymond and Beverly Sackler School of Physics and Astronomy, Tel Aviv University, Israel \\ ${ }^{2}$ Department of Particle Physics, Weizmann Institute of Science, Rehovot 76100, Israel \\ ${ }^{3}$ High Energy Physics Division, Argonne National Laboratory Argonne, IL 60439-4815, USA \\ ${ }^{4}$ Department of Physical Sciences, University of Helsinki, POB 64, FIN-0014 Finland
}

\begin{abstract}
We discuss several highly accurate theoretical predictions for masses of baryons containing the $b$ quark which have been recently confirmed by experimental data. Proper treatment of the color-magnetic hyperfine interaction in QCD is crucial for obtaining these results. Several predictions are given for additional properties of heavy baryons. We also discuss the two charged exotic resonances $Z_{b}$ with quantum numbers of a $(b \bar{b} u \bar{d})$ tetraquark, very recently reported by Belle in the channel $\left[\Upsilon(n S) \pi^{+}, n=1,2,3\right]$. Among possible implications are deeply bound $I=0$ counterparts of the $Z_{b}$-s and existence of a $\Sigma_{b}^{+} \Sigma_{b}^{-}$dibaryon, a beauteron.
\end{abstract}

\section{Introduction}

QCD describes hadrons as valence quarks in a sea of gluons and $\bar{q} q$ pairs. At distances above $\sim 1 \mathrm{GeV}^{-1}$ quarks acquire an effective constituent mass due to chiral symmetry breaking. A hadron can then be thought of as a bound state of constituent quarks. In the zeroth-order approximation the hadron mass $M$ is then given by the sum of the masses of its constituent quarks $m_{i}, M=\sum_{i} m_{i}$. The binding and kinetic energies are "swallowed" by the constituent quarks masses. The first and most important correction comes from the color hyper-fine (HF) chromo-magnetic interaction,

$$
\begin{aligned}
M & =\sum_{i} m_{i}+\sum_{i<j} V_{i j}^{H F(Q C D)} ; \\
V_{i j}^{H F(Q C D)} & =v_{0}\left(\vec{\lambda}_{i} \cdot \vec{\lambda}_{j}\right) \frac{\vec{\sigma}_{i} \cdot \vec{\sigma}_{j}}{m_{i} m_{j}}\left\langle\psi\left|\delta\left(r_{i}-r_{j}\right)\right| \psi\right\rangle
\end{aligned}
$$

where $v_{0}$ gives the overall strength of the HF interaction, $\vec{\lambda}_{i, j}$ are the $S U(3)$ color matrices, $\sigma_{i, j}$ are the quark spin operators and $|\psi\rangle$ is the hadron wave function. This is a contact spin-spin interaction, analogous to the EM hyperfine interaction, which is a product of the magnetic moments, $V_{i j}^{H F(Q E D)} \propto \vec{\mu}_{i} \cdot \vec{\mu}_{j}=e^{2} \vec{\sigma}_{i} \cdot \vec{\sigma}_{j} /\left(m_{i} m_{j}\right)$. In QCD, the $S U(3)_{c}$ generators take place of the electric charge. From eq. (1) many very accurate results have been obtained for the masses of the groundstate hadrons. Nevertheless, several caveats are in order. First, this is a low-energy phenomenological

\footnotetext{
ae-mail: marek@proton.tau.ac.il

be-mail: harry.lipkin@weizmann.ac.il

ce-mail: nils.tornqvist@ helsinki.fi
}

This is an Open Access article distributed under the terms of the Creative Commons Attribution License 2.0, which permits unrestricted use, distribution, and reproduction in any medium, provided the original work is properly cited. 
model, still awaiting a rigorous derivation from QCD. It is far from providing a complete description of the hadronic spectrum, but it provides excellent predictions for mass splittings and magnetic moments. The crucial assumptions of the model are: (a) HF interaction is considered as a perturbation which does not change the wave function; (b) effective masses of quarks are the same inside mesons and baryons; (c) there are no 3-body effects.

\section{Effective masses of quarks}

Constituent quark mass differences depend strongly on the flavor of the spectator or "neighbor" quark [1]. For example, $m_{s}-m_{d} \approx 180 \mathrm{MeV}$ when the spectator is a light quark but the same mass difference is only about $90 \mathrm{MeV}$ when the spectator is a $b$ quark, as shown in Table I.

Since these are effective masses, we should not be surprised that their difference is affected by the environment, but the large size of the shift is quite surprising and its quantitative derivation from QCD is an outstanding challenge for theory.

We can extract the ratio of the constituent quark masses from the ratio of the the hyperfine splittings in the corresponding mesons. The hyperfine splitting between $K^{*}$ and $K$ mesons is given by

$$
M\left(K^{*}\right)-M(K)=v_{0} \frac{\vec{\lambda}_{u} \cdot \vec{\lambda}_{s}}{m_{u} m_{s}}\left[\left(\vec{\sigma}_{u} \cdot \vec{\sigma}_{s}\right)_{K^{*}}-\left(\vec{\sigma}_{u} \cdot \vec{\sigma}_{s}\right)_{K}\right]\langle\psi|\delta(r)| \psi\rangle=4 v_{0} \frac{\vec{\lambda}_{u} \cdot \vec{\lambda}_{s}}{m_{u} m_{s}}\langle\psi|\delta(r)| \psi\rangle,
$$

and similarly for hyperfine splitting between $D^{*}$ and $D$ with $s \rightarrow c$ everywhere. From (2) and its $D$ analogue we then immediately obtain

$$
\frac{M\left(K^{*}\right)-M(K)}{M\left(D^{*}\right)-M(D)} \approx \frac{m_{c}}{m_{s}}
$$

We will now discuss how extend relation (3) to baryons and how to use the extended relation to obtain predictions for masses of heavy baryons containing the $b$-quark.

\subsection{Color hyperfine splitting in baryons}

As an example of hyperfine splitting in baryons, let us now discuss the HF splitting in the $\Sigma$ ( $u d s$ ) baryons. $\Sigma^{*}$ has spin $\frac{3}{2}$, so the $u$ and $d$ quarks must be in a state of relative spin 1 . The $\Sigma$ has isospin 1 , so the wave function of $u$ and $d$ is symmetric in flavor. It is also symmetric in space, since in the ground state the quarks are in a relative $S$-wave. On the other hand, the $u$ - $d$ wave function is antisymmetric in color, since the two quarks must couple to a $3^{*}$ of color to neutralize the color of the third quark. The $u$ - $d$ wave function must be antisymmetric in flavor $\times$ spin $\times$ space $\times$ color, so it follows it must be symmetric in spin, i.e. $u$ and $d$ are coupled to spin one. Since $u$ and $d$ are in spin 1 state in both $\Sigma^{*}$ and $\Sigma$ their HF interaction with each other cancels between the two and thus the $u$ - $d$ pair does not contribute to the $\Sigma^{*}-\Sigma$ HF splitting,

$$
M\left(\Sigma^{*}\right)-M(\Sigma)=6 v_{0} \frac{\vec{\lambda}_{u} \cdot \vec{\lambda}_{s}}{m_{u} m_{s}}\left\langle\psi\left|\delta\left(r_{r s}\right)\right| \psi\right\rangle
$$




\begin{tabular}{|c|c|c|c|c|c|c|c|c|}
\hline \multirow{3}{*}{ observable } & \multirow{2}{*}{\multicolumn{2}{|c|}{ baryons }} & \multicolumn{4}{|c|}{ mesons } & \multirow{3}{*}{$\begin{array}{c}\Delta m_{\text {Bar }} \\
\mathrm{MeV}\end{array}$} & \multirow{3}{*}{$\begin{array}{c}\Delta m_{M e s} \\
\mathrm{MeV}\end{array}$} \\
\hline & & & \multicolumn{2}{|c|}{$J=1$} & \multicolumn{2}{|c|}{$J=0$} & & \\
\hline & $B_{i}$ & $B_{j}$ & $\mathcal{V}_{i}$ & $\mathcal{V}_{j}$ & $\mathcal{P}_{i}$ & $\mathcal{P}_{j}$ & & \\
\hline \multirow{2}{*}{$\left\langle m_{s}-m_{u}\right\rangle_{d}$} & sud & uud & $s \bar{d}$ & $u \bar{d}$ & $s \bar{d}$ & $u \bar{d}$ & \multirow[t]{2}{*}{177} & \multirow[t]{2}{*}{179} \\
\hline & $\Lambda$ & $N$ & $K^{*}$ & $\rho$ & $K$ & $\pi$ & & \\
\hline \multirow{2}{*}{$\left\langle m_{s}-m_{u}\right\rangle_{c}$} & & & $c \bar{s}$ & $c \bar{u}$ & $c \bar{s}$ & $c \bar{u}$ & & \multirow{2}{*}{103} \\
\hline & & & $D_{s}^{*}$ & $D_{s}^{*}$ & $D_{s}$ & $D_{s}$ & & \\
\hline \multirow{2}{*}{$\left\langle m_{s}-m_{u}\right\rangle_{b}$} & & & $b \bar{s}$ & $b \bar{u}$ & $b \bar{s}$ & $b \bar{u}$ & & \multirow[t]{2}{*}{91} \\
\hline & & & $B_{s}^{*}$ & $B_{s}^{*}$ & $B_{s}$ & $B_{s}$ & & \\
\hline \multirow[t]{2}{*}{$\left\langle m_{c}-m_{u}\right\rangle_{d}$} & cud & uud & $c \bar{d}$ & $u \bar{d}$ & $c \bar{d}$ & $u \bar{d}$ & \multirow[t]{2}{*}{1346} & \multirow[t]{2}{*}{1360} \\
\hline & $\Lambda_{c}$ & $N$ & $D^{*}$ & $\rho$ & $D$ & $\pi$ & & \\
\hline \multirow{2}{*}{$\left\langle m_{c}-m_{u}\right\rangle_{c}$} & & & $c \bar{c}$ & $u \bar{c}$ & $c \bar{c}$ & $u \bar{c}$ & & \multirow[t]{2}{*}{1095} \\
\hline & & & $\psi$ & $D^{*}$ & $\eta_{c}$ & $D$ & & \\
\hline \multirow[t]{2}{*}{$\mid\left\langle m_{c}-m_{s}\right\rangle_{d}$} & cud & sud & $c \bar{d}$ & $s \bar{d}$ & $c \bar{d}$ & $s \bar{d}$ & \multirow[t]{2}{*}{1169} & \multirow[t]{2}{*}{1180} \\
\hline & $\Lambda_{c}$ & $\Lambda$ & $D^{*}$ & $K^{*}$ & $D$ & $K$ & & \\
\hline \multirow[t]{2}{*}{$\left\langle m_{c}-m_{s}\right\rangle_{c}$} & & & $c \bar{c}$ & $s \bar{c}$ & $c \bar{c}$ & $s \bar{c}$ & & \multirow[t]{2}{*}{991} \\
\hline & & & $\psi$ & $D_{s}^{*}$ & $\eta_{c}$ & $D_{s}$ & & \\
\hline
\end{tabular}

\begin{tabular}{|c||c|c||c|c|c|c||c|c|}
\hline$\left\langle m_{b}-m_{u}\right\rangle_{d}$ & bud & $u u d$ & $b \bar{d}$ & $u \bar{d}$ & $b \bar{d}$ & $u \bar{d}$ & 4685 & 4700 \\
\cline { 2 - 7 } & $\Lambda_{b}$ & $N$ & $B^{*}$ & $\rho$ & $B$ & $\pi$ & & \\
\hline \hline$\left\langle m_{b}-m_{u}\right\rangle_{s}$ & & & $b \bar{s}$ & $u \bar{s}$ & $b \bar{s}$ & $u \bar{s}$ & & 4613 \\
\cline { 2 - 7 } & & & $B_{s}^{*}$ & $K^{*}$ & $B_{s}$ & $K$ & & \\
\hline
\end{tabular}

\begin{tabular}{|c||c|c||c|c|c|c||c|c|}
\hline \multirow{2}{*}{$\left\langle m_{b}-m_{s}\right\rangle_{d}$} & $b u d$ & $s u d$ & $b \bar{d}$ & $s \bar{d}$ & $b \bar{d}$ & $s \bar{d}$ & 4508 & 4521 \\
\cline { 2 - 7 } & $\Lambda_{b}$ & $\Lambda$ & $B^{*}$ & $K^{*}$ & $B$ & $K$ & & \\
\hline
\end{tabular}

\begin{tabular}{|c||c|c||c|c|c|c||c|c|}
\hline \multirow{2}{*}{$\left\langle m_{b}-m_{c}\right\rangle_{d}$} & $b u d$ & $s u d$ & $b \bar{d}$ & $c \bar{d}$ & $b \bar{d}$ & $c \bar{d}$ & \multirow{2}{*}{3339} & 3341 \\
\cline { 2 - 7 } & $\Lambda_{b}$ & $\Lambda_{c}$ & $B^{*}$ & $D^{*}$ & $B$ & $D$ & & \\
\hline \hline$\left\langle m_{b}-m_{c}\right\rangle_{s}$ & & & $b \bar{s}$ & $c \bar{s}$ & $b \bar{s}$ & $c \bar{s}$ & & \multirow{2}{*}{3328} \\
\cline { 2 - 7 } & & & $B_{s}^{*}$ & $D_{s}^{*}$ & $B_{s}$ & $D_{s}$ & & \\
\hline
\end{tabular}

Table I. Differences of effective quark masses [1]. The mass difference between two quarks of different flavors are seen to have the same value to a good approximation when they are bound to a nonstrange antiquark to make a meson and bound to a nonstrange diquark to make a baryon. 
we can then use eqs. (2) and (4) to compare the quark mass ratio obtained from mesons and baryons:

$$
\begin{array}{ll}
\left(\frac{m_{c}}{m_{s}}\right)_{\text {Bar }}=\frac{M_{\Sigma^{*}}-M_{\Sigma}}{M_{\Sigma_{c}^{*}}-M_{\Sigma_{c}}}=2.84 ; & \left(\frac{m_{c}}{m_{s}}\right)_{M e s}=\frac{M_{K^{*}}-M_{K}}{M_{D^{*}}-M_{D}}=2.81 \\
\left(\frac{m_{c}}{m_{u}}\right)_{\text {Bar }}=\frac{M_{\Delta}-M_{p}}{M_{\Sigma_{c}^{*}}-M_{\Sigma_{c}}}=4.36 ; & \left(\frac{m_{c}}{m_{u}}\right)_{\text {Mes }}=\frac{M_{\rho}-M_{\pi}}{M_{D^{*}}-M_{D}}=4.46
\end{array}
$$

We find the same value from mesons and baryons $\pm 2 \%$.

The presence of a fourth flavor gives us the possibility of obtaining a new type of mass relation between mesons and baryons. The $\Sigma-\Lambda$ mass difference is believed to be due to the difference between the $u-d$ and $u-s$ hyperfine interactions. Similarly, the $\Sigma_{c}-\Lambda_{c}$ mass difference is believed to be due to the difference between the $u-d$ and $u-c$ hyperfine interactions. We therefore obtain the relation

$$
\left(\frac{\frac{1}{m_{u}^{2}}-\frac{1}{m_{u} m_{c}}}{\frac{1}{m_{u}^{2}}-\frac{1}{m_{u} m_{s}}}\right)_{\text {Bar } / \text { Mes }}=\frac{M_{\Sigma_{c}}-M_{\Lambda_{c}}}{M_{\Sigma}-M_{\Lambda}}=2.16 \approx \frac{\left(M_{\rho}-M_{\pi}\right)-\left(M_{D^{*}}-M_{D}\right)}{\left(M_{\rho}-M_{\pi}\right)-\left(M_{K^{*}}-M_{K}\right)}=2.10
$$

The meson and baryon relations agree to $\pm 3 \%$.

We can write down an analogous relation for hadrons containing the $b$ quark instead of the $s$ quark, obtaining the prediction for splitting between $\Sigma_{b}$ and $\Lambda_{b}$ :

$$
\frac{M_{\Sigma_{b}}-M_{\Lambda_{b}}}{M_{\Sigma}-M_{\Lambda}}=\frac{\left(M_{\rho}-M_{\pi}\right)-\left(M_{B^{*}}-M_{B}\right)}{\left(M_{\rho}-M_{\pi}\right)-\left(M_{K^{*}}-M_{K}\right)}=2.51
$$

yielding $M\left(\Sigma_{b}\right)-M\left(\Lambda_{b}\right)=194 \mathrm{MeV}[1,2]$. This splitting was measured by CDF [3], with isospinaveraged mass difference $M\left(\Sigma_{b}\right)-M\left(\Lambda_{b}\right)=192 \mathrm{MeV}$.

There is also the prediction for the spin splittings, good to $5 \%$

$$
M\left(\Sigma_{b}^{*}\right)-M\left(\Sigma_{b}\right)=\frac{M\left(B^{*}\right)-M(B)}{M\left(K^{*}\right)-M(K)} \cdot\left[M\left(\Sigma^{*}\right)-M(\Sigma)\right]=22 \mathrm{MeV}
$$

to be compared with $21 \mathrm{MeV}$ from the isospin-average of CDF measurements [3]. The challenge is to understand how and under what assumptions one can derive from QCD the very simple model of hadronic structure at low energies which leads to such accurate predictions.

\section{Magnetic Moments of Heavy Quark Baryons}

In $\Lambda, \Lambda_{c}$ and $\Lambda_{b}$ baryons the light quarks are coupled to spin zero. Therefore the magnetic moments of these baryons are determined by the magnetic moments of the $s, c$ and $b$ quarks, respectively. The latter are proportional to the chromomagnetic moments which determine the hyperfine splitting in baryon spectra. We can use this fact to predict the $\Lambda_{c}$ and $\Lambda_{b}$ baryon magnetic moments by relating 
them to the hyperfine splittings in the same way as given in the original prediction [5] of the $\Lambda$ magnetic moment. We obtain

$$
\begin{aligned}
& \mu_{\Lambda_{c}}=-2 \mu_{\Lambda} \cdot \frac{M_{\Sigma_{c}^{*}}-M_{\Sigma_{c}}}{M_{\Sigma^{*}}-M_{\Sigma}}=0.43 \text { n.m.; } \\
& \mu_{\Lambda_{b}}=\mu_{\Lambda} \cdot \frac{M_{\Sigma_{b}^{*}}-M_{\Sigma_{b}}}{M_{\Sigma^{*}}-M_{\Sigma}}=-0.067 \text { n.m. }
\end{aligned}
$$

We hope these observables can be measured in foreseeable future and view the predictions (10) as a challenge for the experimental community.

\section{Predicting the Masses of b-Baryons}

On top of the already discussed $\Sigma_{b}$ with quark content $b q q, q=u, d$. there are two additional groundstate $b$-baryons, $\Xi_{b}$ and $\Omega_{b}$.

\section{$\Xi_{b}$ mass}

The quark content of $\Xi_{b}$ is $b s q$. $\Xi_{b}$ can be obtained from an "ordinary" $\Xi$ ( $s s d$ or $s s u$ ) by replacing one of the $s$ quarks by a $b$, with one important difference. In the ordinary $\Xi$, Fermi statistics dictates that two $s$ quarks must couple to spin-1, while in the ground state of $\Xi_{b}$ the $(s q)$ diquarks have spin zero. Consequently, the $\Xi_{b}$ mass is given by the expression: $\Xi_{b}=m_{b}+m_{s}+m_{u}-3 v\left\langle\delta\left(r_{u s}\right)\right\rangle / m_{u} m_{s}$. The $\Xi_{b}$ mass can thus be predicted using the known $\Xi_{c}$ baryon mass as a starting point and adding the corrections due to mass differences and HF interactions:

$$
\Xi_{b}=\Xi_{c}+\left(m_{b}-m_{c}\right)-3 v\left(\left\langle\delta\left(r_{u s}\right)\right\rangle_{\Xi_{b}}-\left\langle\delta\left(r_{u s}\right)\right\rangle_{\Xi_{c}}\right) /\left(m_{u} m_{s}\right)
$$

Since the $\Xi_{b}$ and $\Xi_{c}$ baryons contain a strange quark, and the effective constituent quark masses depend on the spectator quark, the optimal way to estimate the mass difference $\left(m_{b}-m_{c}\right)$ is from mesons which contain both $s$ and $b$ or $c$ quarks:

$$
m_{b}-m_{c}=\frac{1}{4}\left(3 B_{s}^{*}+B_{s}\right)-\frac{1}{4}\left(3 D_{s}^{*}+D_{s}\right)=3324.6 \pm 1.4 \mathrm{MeV}
$$

On this basis we predicted [7] $M\left(\Xi_{b}\right)=5795 \pm 5 \mathrm{MeV}$. Our paper was submitted on June 14, 2007. The next day CDF announced the result [9], $M\left(\Xi_{b}\right)=5792.9 \pm 2.5 \pm 1.7 \mathrm{MeV}$, following up on an earlier D0 measurement, $M\left(\Xi_{b}\right)=5774 \pm 11 \pm 15 \mathrm{MeV}[8]$.

In November $2011 \mathrm{CDF}$ discovered $\Xi_{b}^{0}$. i.e. $(u s b)$, the neutral partner of the $\Xi_{b}^{-}(d s b)$ with mass $5787.8 \pm 5.0$ (stat.) \pm 1.3 (sys.) $\mathrm{MeV}$ [10], to be compared with our prediction $5786.7 \pm 3.0 \mathrm{MeV}$ [13]

In early $2012 \mathrm{LHCb}$ provided an independent measurement of $\Xi_{b}^{-}$mass: $5796.5 \pm 1.2 \pm 1.2 \mathrm{MeV}$ [11], in excellent agreement with the CDF results and with our theoretical predictions.

In April $2012 \mathrm{CDF}$ discovered a new, excited $\Xi_{b}$ baryon decaying into $\Xi_{b}^{-} \pi^{+}$with the mass $5945.0 \pm 0.7$ (stat.) \pm 0.3 (sys.) \pm 2.7 (PDG) $\mathrm{MeV}[12]$. The ground-state $\Xi_{b}(q s b)$ has spin $1 / 2$ with 
$q s$ coupled to spin 0 . There are two excited states: one is called $\Xi_{b}^{\prime}$ and has spin $1 / 2$ with $q s$ coupled to spin 1 . The other is called $\Xi_{b}^{*}$ and has spin $3 / 2$. CMS has not measured the spin of the excited $\Xi_{b}$ baryon, therefore a priori it could be either $\Xi_{b}^{\prime}$ or $\Xi_{b}^{*}$. Interestingly enough, CMS relied on theoretical work to identify the excited baryon as $\Xi_{b}^{*}$, noting that according to the theoretical predictions $\Xi_{b}^{\prime}$ is expected to lie below the $\Xi_{b}^{-} \pi^{+}$threshold. The mass of $\Xi_{b}^{*}$ is close to our prediction $5959 \pm 4 \mathrm{MeV}$ [13].

\section{$\Omega_{b}$ mass}

For the spin-averaged $\Omega_{b}$ mass we have

$$
\begin{aligned}
\frac{1}{3}\left(2 M\left(\Omega_{b}^{*}\right)+M\left(\Omega_{b}\right)\right) & =\frac{1}{3}\left(2 M\left(\Omega_{c}^{*}\right)+M\left(\Omega_{c}\right)\right)+\left(m_{b}-m_{c}\right)_{B_{s}-D_{s}} \\
& =6068.9 \pm 2.4 \mathrm{MeV}
\end{aligned}
$$

For the HF splitting we obtain

$$
\begin{aligned}
M\left(\Omega_{b}^{*}\right)-M\left(\Omega_{b}\right) & =\left(M\left(\Omega_{c}^{*}\right)-M\left(\Omega_{c}\right)\right) \frac{m_{c}}{m_{b}} \frac{\left\langle\delta\left(r_{b s}\right)\right\rangle_{\Omega_{b}}}{\left\langle\delta\left(r_{c s}\right)\right\rangle_{\Omega_{c}}} \\
& =30.7 \pm 1.3 \mathrm{MeV}
\end{aligned}
$$

leading to the following predictions:

$$
M\left(\Omega_{b}\right)=6052.1 \pm 5.6 \mathrm{MeV} ; M\left(\Omega_{b}^{*}\right)=6082.8 \pm 5.6 \mathrm{MeV}
$$

About four months after our prediction (15) for $\Omega_{b}$ mass [13], D0 collaboration published the first measurement of $\Omega_{b}$ mass [14]: $M\left(\Omega_{b}\right)_{D 0}=6165 \pm 10$ (stat. $) \pm 13$ (syst.) $\mathrm{MeV}$. The deviation from the central value of our prediction was huge, $113 \mathrm{MeV}$. Understandably, we were very eager to see the CDF result. CDF published their result about nine months later, in May 2009 [15]: $M\left(\Omega_{b}\right)_{C D F}=$ $6054 \pm 6.8$ (stat. $) \pm 0.9$ (syst. $) \mathrm{MeV}$.

The CDF result for $\Omega_{b}$ mass was confirmed in early 2012 by an independent measurement from $\mathrm{LHCb}[11]: M\left(\Omega_{b}\right)_{L H C b}=6050.3 \pm 4.5 \pm 2.2$.

Fig. 1 shows a comparison of our predictions for the masses of $\Sigma_{b}, \Xi_{b}$ and $\Omega_{b}$ baryons with the experimental data from CDF, $\mathrm{LHCb}$ and CMS.

We have made additional predictions $[7,13]$ for some excited states of $b$-baryons. Our results are summarized in Table 10 of Ref. [13].

The sign in our prediction $M\left(\Sigma_{b}^{*}\right)-M\left(\Sigma_{b}\right)<M\left(\Omega_{b}^{*}\right)-M\left(\Omega_{b}\right)$, appears to be counterintuitive, since the color hyperfine interaction is inversely proportional to the quark mass. This reversed inequality is not predicted by other recent approaches [16-18], but it is also seen in the charm data, $M\left(\Sigma_{c}^{*}\right)-M\left(\Sigma_{c}\right)=$ $64.3 \pm 0.5 \mathrm{MeV}<M\left(\Omega_{c}^{*}\right)-M\left(\Omega_{c}\right)=70.8 \pm 1.5 \mathrm{MeV}$. This suggests that the sign of the $S U(3)$ symmetry breaking gives information about the form of the potential. It is of interest to follow this clue theoretically and experimentally.

\section{Heavy exotics}

Ordinary hadrons contain either a $q \bar{q}$ pair or 3 quarks. The possible color representations of quark combinations are then completely determined by confinement. In a meson the $q \bar{q}$ pair must couple to 
ICFP 2012

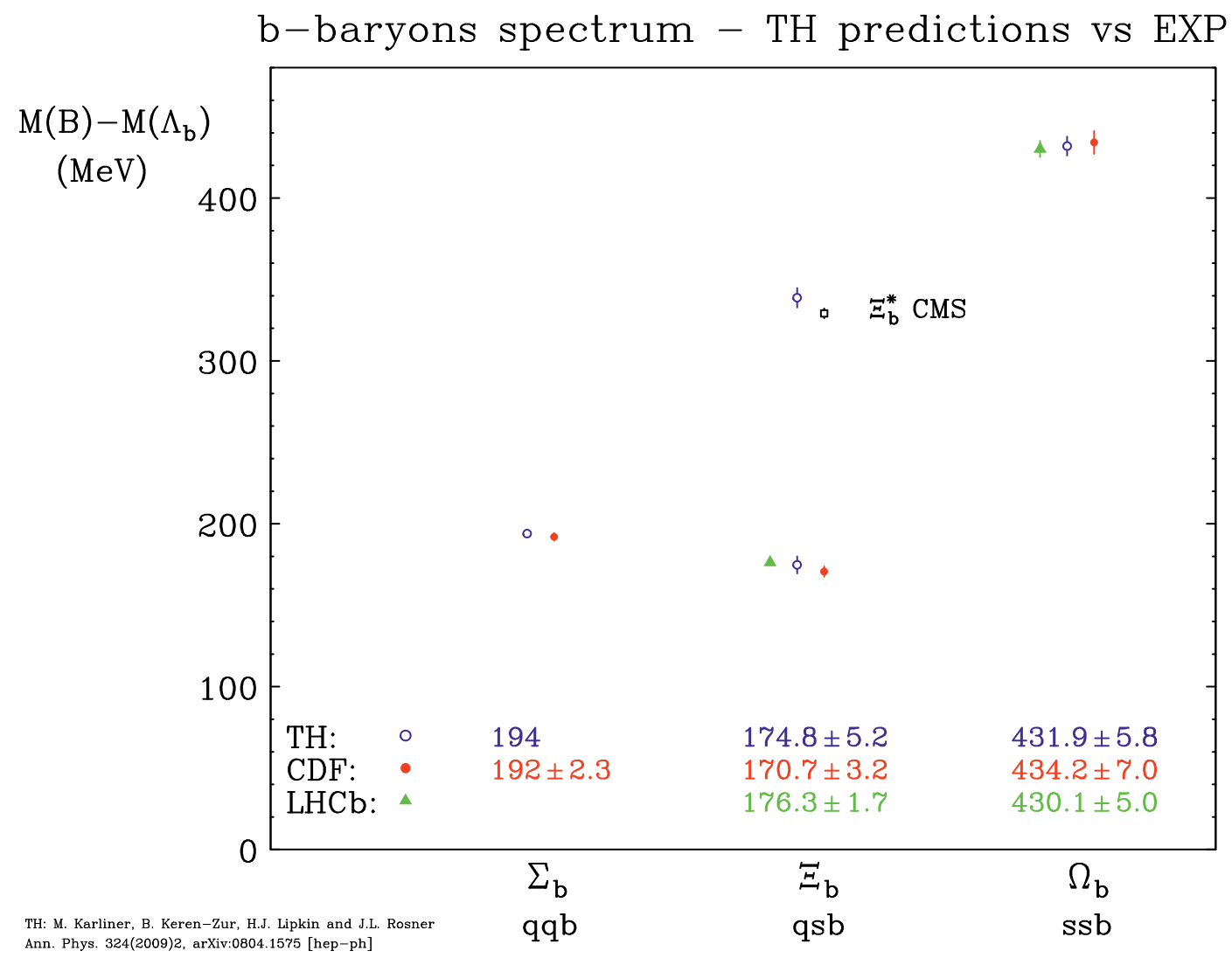

Figure 1. Masses of $b$-baryons - theoretical predictions [7, 13] vs. experiment.

a color singlet and in a baryon any two quarks must couple to an anti-triplet of color, to neutralize the color charge of the third quark. The situation is very different in exotic hadrons which contain both $q q$ and $q \bar{q}$ pairs, eg. a tetraquark with two heavy quarks $Q$ and two light quarks $q, Q \bar{Q} q \bar{q}$. Such states have important color-space correlations that are completely absent in ordinary mesons and baryons [19]. One also needs to keep in mind that the $q-\bar{q}$ interaction is much stronger than $q-q$ interaction. The result is emergence of color structures that are totally different from those in normal hadrons. In turn, this leads to some very unusual experimental properties of such states. Until May 2011 the leading candidate has been the $X(3872)$, which is most likely either a $c \bar{c} q \bar{q}$ or a threshold bound state of $D$ and $\bar{D}^{*}$. Given that $X(3872)$ exists, it is fascinating to explore possible analogues containing $b$ quarks. General considerations suggest that such states should be more strongly bound, since the attraction due to color forces is the roughly same, but the repulsion due to kinetic energy is smaller, as $E_{k} \sim p^{2} / m_{Q}$. Using a simple model, we have suggested that $b \bar{b} q \bar{q}$ might be below the $B \bar{B}$ threshold and $b \bar{c} q \bar{q}$ might be below the $B \bar{D}$ threshold. A crucial difference vs. ordinary mesons is that $(Q q)(\bar{Q} \bar{q})$ 

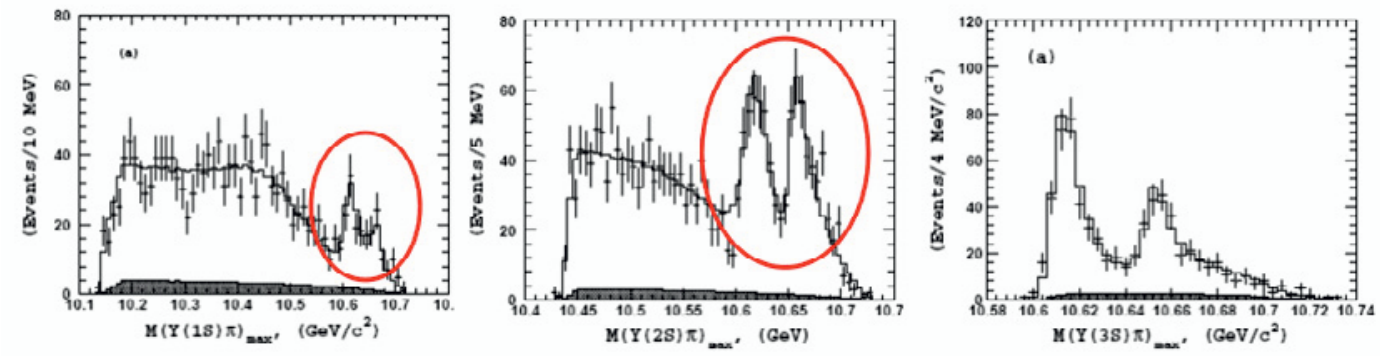

Figure 2. Invariant mass of the $\Upsilon(n) \pi$ systems, $n=1,2,3$, Ref. [22].

can form a $\overline{\mathbf{6}} \mathbf{6}$ color configuration which has much stronger binding than $\overline{\mathbf{3} 3}$. Some of these states have exotic electric charge, e.g. $b d \bar{c} \bar{u} \rightarrow J / \psi \pi^{-} \pi^{-}$. Their decays have striking experimental signatures: monoenergetic photons and/or pions, e.g. $b q \bar{c} \bar{q}$ with $I=0$ above $B_{c} \pi$ threshold can decay into $B_{c} \pi$ via isospin violation, or electromagnetically into $B_{c} \gamma$, both very narrow.

Hadrons containing two $b$ quarks, such as double-bottom baryons $b b q$ or $b \bar{b} q \bar{q}$ and $b b \bar{q} \bar{q}$ tetraquarks have a unique and a spectacular decay mode with two $J / \psi$-s in the final state. To see this, recall that a $b$ quark can decay via the hadronic mode $b \rightarrow \bar{c} c s \rightarrow J / \psi s$. If both $b$ quarks in a double-bottom hadron decay this way, for a $b b$ baryon we get $(b b q) \rightarrow J / \psi J / \psi(s s q) \rightarrow J / \psi J / \psi \Xi$, and similarly for a tetraquark: $(b \bar{b} q \bar{q}) \rightarrow J / \psi J / \psi(\bar{s} s \bar{q} q) \rightarrow J / \psi J / \psi K K$, etc., with all final state hadrons coming from the same vertex. This unique signature is however hampered by a very low rate expected for such a process, especially if one uses dimuons to identify the $J / \psi$-s. It is both challenge and a opportunity for $\mathrm{LHCb}[19]$.

\section{Exotic double-bottom hadrons $Z_{b}$ : theoretical prediction and discovery by Belle}

In 2008 Belle reported [20] anomalously large (by two orders of magnitude) branching ratios for the decays $\Upsilon(5 S) \rightarrow \Upsilon(m S) \pi^{+} \pi^{-}, \quad m=1,2$. In [21] we suggested that the enhancement is due to an intermediate state of a tetraquark $T_{\bar{b} b}=(\bar{b} b u \bar{d})$ and a pion, mediating the two-step process

$$
\Upsilon(5 S) \rightarrow T_{\bar{b} b}^{ \pm} \pi^{\mp} \rightarrow \Upsilon(m S) \pi^{+} \pi^{-}
$$

We proposed looking for the $(\bar{b} b u \bar{d})$ tetraquark in these decays as peaks in the invariant mass of $\Upsilon(1 S) \pi^{+}$or $\Upsilon(2 S) \pi^{+}$systems.

Very recently Belle collaboration confirmed this prediction, announcing [22] the observation of two charged bottomonium-like resonances $Z_{b}$ as narrow structures in $\pi^{ \pm} \Upsilon(n S)(n=1,2,3)$ and $\pi^{ \pm} h_{b}(m P)(m=1,2)$ mass spectra that are produced in association with a single charged pion in $\Upsilon(5 S)$ decays, cf. Fig. 2.

The measured masses of the two structures averaged over the five final states are $M_{1}=10608.4 \pm 2.0 \mathrm{MeV}, M_{2}=10653.2 \pm 1.5 \mathrm{MeV}$, both with a width of about $15 \mathrm{MeV}$.

Interestingly enough, the two masses $M_{1}$ and $M_{2}$ are about $3 \mathrm{MeV}$ above the respective $B^{*} \bar{B}$ and $B^{*} \bar{B}^{*}$ thresholds, cf. Fig. 3 .

This strongly suggests a parallel with $X(3872)$, whose mass is almost exactly at the $D^{*} \bar{D}$ threshold. It also raises the possibility that such states might have a complementary description as deuteron-like "molecule" of two heavy mesons quasi-bound by pion exchange [23, 24]. 


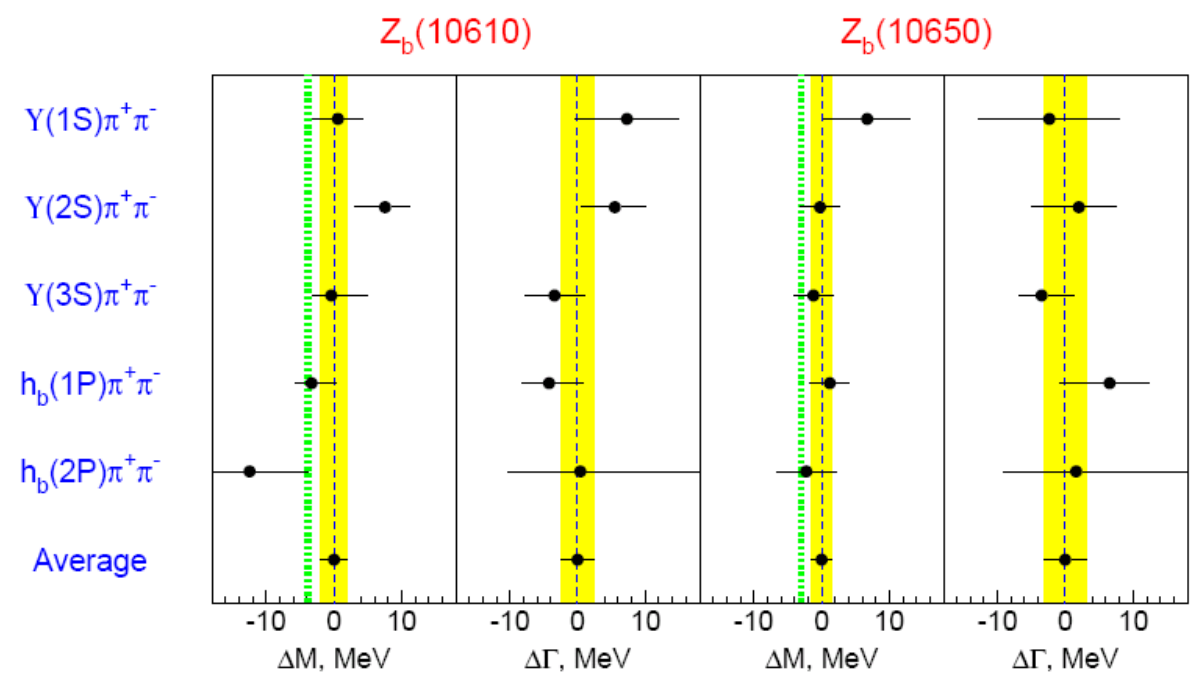

Figure 3. Comparison of $Z_{b}(10610)$ and $Z_{b}(10650)$ parameters obtained from five different decay channels [22]. The vertical lines consisting of horizontal dashes indicate the $B^{*} \bar{B}$ and $B^{*} \bar{B}^{*}$ thresholds, respectively. $\Delta M$ and $\Delta \Gamma$ denote the deviation of each experiment from the average value of the mass and the width, respectively.

The attraction due to $\pi$ exchange is 3 times weaker in the $I=1$ channel than in the $I=0$ channel. This is because for $I=1$ only $\pi^{0}$ contributes, whereas for $I=0$ both $\pi^{0}$ and $\pi^{ \pm}$contribute. Consequently, in the charm system the $I=1$ state is far above the $D^{*} \bar{D}$ threshold and only the $I=0$ $X(3872)$ is bound $2 \mathrm{MeV}$ below the average of the isospin-related $D^{+} D^{*-}$ and $D^{0} \bar{D}^{0}$ thresholds. The situation is likely to be different in the bottom system. This is because the attraction due to $\pi$ exchange is essentially the same, but the $B$ mesons are much heavier than $D$ mesons, so the kinetic energy is much smaller by a factor of $\sim m(B) / m(D) \approx 2.8$. Therefore the net binding is much stronger than in the charm system. This raises two very interesting possibilities:

1. the $Z_{b}$ states are virtually bound $S$-wave $B^{*} \bar{B}$ and $B^{*} \bar{B}^{*}$ states, i.e. states which analytically are second sheet poles just below threshold, but which appear as standard Breit-Wigner resonances slightly above threshold; see e.g. [25]. The quantum numbers of these states are $I=1, J^{P}=1^{+}$. The neutral members of their isomultiplets have $C=-1, G=+1$.

2. since the binding in the $I=0$ channel is much stronger than in the $I=1$ channel, if we neglect effects other than $\pi$ exchange we expect the corresponding $I^{G}=0^{+}, J^{P C}=1^{++}$states to be up to 40-50 MeV below the thresholds [26]. The $I=0$ states would then be expected close in mass to the $\Upsilon(4 S)$. Their expected decay modes are

$$
Z_{b}(I=0) \rightarrow \Upsilon(m S) \pi^{+} \pi^{-} \quad \text { and } \quad Z_{b}(I=0) \rightarrow \Upsilon(m S) \gamma
$$

as well as

$$
Z_{b}(I=0) \rightarrow B \bar{B} \gamma \quad \text { via } \quad B^{*} \rightarrow B \gamma, \quad E_{\gamma}=46 \mathrm{MeV} ;
$$

which might well be within the reach of $\mathrm{LHCb}$. 


\section{A $\left(\Sigma_{b}^{+} \Sigma_{b}^{-}\right)$beauteron dibaryon?}

The discovery of the $Z_{b}$ states and their probable interpretation as $B^{*} \bar{B}$ and $B^{*} \bar{B}^{*}$ bound by pion exchange raises an interesting possibility that a strongly bound $\Sigma_{b}^{+} \Sigma_{b}^{-}$deuteron-like state might exist, a beauteron.

The reasoning behind this is as follows. The existence of the $Z_{b}$ as quasi-bound states shows that the $\pi$-mediated attraction between the heavy $B$-mesons is quite strong. The net attraction results from a competition between the pion-induced attraction and repulsion due to kinetic energy and possibly also due to other meson exchanges. The kinetic energy in the $B \bar{B}^{*}$ and $\Sigma_{b} \Sigma_{b}$ system is small compared to the rest mass of the hadrons, and therefore scales like $p^{2} / \mu_{R E D}$ where $\mu_{R E D}$ is the reduced mass of the hadrons.

The $\Sigma_{b}$ is about $500 \mathrm{MeV}$ heavier than $B^{*}$ and therefore in the $\Sigma_{b} \Sigma_{b}$ system the repulsion due to kinetic energy is significantly smaller than in the $B \bar{B}^{*}$ or $B^{*} \bar{B}^{*}$ system.

In addition, since $\Sigma_{b}$ has $I=1$, it couples more strongly to pions than $B$ and $B^{*}$ which have $I=\frac{1}{2}$. The opposite electric charges of $\Sigma_{b}^{+}$and $\Sigma_{b}^{-}$provide an additional attraction. The upshot is that if $Z_{b}$ indeed are quasi-bound states of $B \bar{B}^{*}$ and $B^{*} \bar{B}^{*}$, the analogous but significantly stronger attraction in the $\Sigma_{b}^{+} \Sigma_{b}^{-}$system could well be sufficient to form a bona-fide bound state. A possible tricky issue is that the $\Sigma_{b}$ baryons themselves decay strongly into $\Lambda_{b} \pi$, with a width of a few MeV. If the $\Sigma_{b}^{+} \Sigma_{b}^{-}$ binding is significantly more than this, the dibaryon bound state can be sufficiently long-lived to be observed experimentally.

A possible decay mode of the beauteron is

$$
\left(\Sigma_{b}^{+} \Sigma_{b}^{-}\right) \rightarrow \Lambda_{b} \Lambda_{b} \pi^{+} \pi^{-}
$$

which might be observable in LHCb. If the beauteron exists, it should also be seen in lattice QCD.

\section{Acknowledgements}

The work on heavy baryons described here was done in collaboration with B. Keren-Zur and J. Rosner. It was supported in part by a grant from the Israel Science Foundation. The research of H.J.L. was supported in part by the U.S. Department of Energy, Division of High Energy Physics, Contract DEAC02-06CH11357.

\section{References}

[1] M. Karliner and H.J. Lipkin,hep-ph/0307243, Phys. Lett. B575 (2003) 249.

[2] M. Karliner and H. J. Lipkin, Phys. Lett. B 660, 539 (2008) [arXiv:hep-ph/0611306].

[3] T. Aaltonen et al. [CDF Collaboration], Phys. Rev. Lett. 99 (2007) 202001.

[4] M. Karliner and H. J. Lipkin, Phys. Lett. B 650, 185 (2007) [arXiv:hep-ph/0608004].

[5] A. De Rujula, H. Georgi and S.L. Glashow, Phys. Rev. D12 (1975) 147

[6] B. Keren-Zur, Annals Phys. 323, 631 (2008) [arXiv:hep-ph/0703011].

[7] M. Karliner, B. Keren-Zur, H. J. Lipkin and J. L. Rosner, arXiv:0706.2163v1 [hep-ph].

[8] V. M. Abazov et al. [D0 Collaboration], Phys. Rev. Lett. 99 (2007) 052001. 
[9] T. Aaltonen et al. [CDF Collaboration], Phys. Rev. Lett. 99 (2007) 052002.

[10] T. Aaltonen et al. [CDF Collaboration], Phys. Rev. Lett. 107 (2011) 102001 [arXiv:1107.4015 [hep-ex]].

[11] D. Milanes [LHCb Collaboration], EPJ Web Conf. 28 (2012) 04010 [arXiv:1201.4717 [hep-ex]].

[12] S. Chatrchyan et al. [CMS Collaboration], Phys. Rev. Lett. 108 (2012) 252002 [arXiv:1204.5955 [hep-ex]].

[13] M. Karliner, B. Keren-Zur, H. J. Lipkin and J. L. Rosner, arXiv:0708.4027 [hep-ph] (unpublished) and arXiv:0804.1575 [hep-ph], Annals Phys 324,2 (2009).

[14] V. M. Abazov et al. [D0 Collaboration], Phys. Rev. Lett. 101, 232002 (2008) [arXiv:0808.4142 [hep-ex]].

[15] T. Aaltonen et al. [CDF Collaboration], Phys. Rev. D 80, 072003 (2009) [arXiv:0905.3123 [hepex]].

[16] D. Ebert et al., Phys. Rev. D 72 (2005) 034026; Phys. Lett. B 659 (2008) 612.

[17] W. Roberts and M. Pervin, Int. J. Mod. Phys. A 23 (2008) 2817 [arXiv:0711.2492 [nucl-th]].

[18] E. E. Jenkins, Phys. Rev. D 77 (2008) 034012.

[19] M. Karliner and H. J. Lipkin, Phys. Lett. B 638, 221 (2006) [arXiv:hep-ph/0601193].

[20] K. F. Chen et al. [Belle Collaboration], Phys. Rev. Lett. 100, 112001 (2008) [arXiv:0710.2577 [hep-ex]].

[21] M. Karliner and H. J. Lipkin, arXiv:0802.0649 [hep-ph].

[22] I. Adachi et al. [Belle Collaboration], arXiv:1105.4583 [hep-ex].

[23] N. A. Törnqvist, Z. Phys. C 61, 525 (1994) [arXiv:hep-ph/9310247]; Phys. Lett. B 590, 209 (2004) [arXiv:hep-ph/0402237].

[24] C. E. Thomas, F. E. Close, Phys. Rev. D78, 034007 (2008). [arXiv:0805.3653 [hep-ph]].

[25] N. A. Tornqvist, Phys. Rev. D 51 (1995) 5312 [hep-ph/9403234].

[26] M. Karliner, H.J. Lipkin and N. A. Törnqvist, unpublished and arXiv:1109.3472 [hep-ph]. 